\title{
An automated identification system for use with computer-controlled tasks
}

\author{
MICHAEL W. ANDREWS \\ State University of New York Health Science Center, Brooklyn, New York
}

\begin{abstract}
Monkeys of various species can use a joystick to perform a variety of computer-generated tasks. The purpose of this paper is to describe an automated system that determines which subject in a social group has completed a task. The system is based on a microchip that is easily injected into the forearm. The present paper demonstrates the technique with 1 animal that had a different chip in each arm.
\end{abstract}

Four recent articles in this journal have attested to the power and flexibility of the Language Research Center's Computerized Test System (Washburn, 1990, 1992; Washburn, Hopkins, \& Rumbaugh, 1989; Washburn \& Rumbaugh, 1992), a computer-controlled, joystick-based system for use with primates. Typically, a monkey uses the joystick to move the cursor on a computer monitor to one or more experimenter-defined target areas. Targets may differ in size, shape, and color and may be moving or stationary. Tasks that have been employed include pursuit, matching to sample, sameness-difference discriminations, and maze solving.

Versions of the joystick tasks have been adapted for use in the Primate Behavior Laboratory at the SUNY Health Science Center (e.g., Andrews, 1993; Andrews \& Rosenblum, in press; Rosenblum \& Andrews, in press). A major thrust of the work has been the application of joystick tasks in social group settings with 9- to 24-h access to the tasks. Thus far, the tasks have been used in social groups of bonnet macaques (Macaca radiata), pigtail macaques (Macaca nemestrina), and squirrel monkeys (Saimiri sciureus). Significant problems relating to application of the tasks in a social setting include identification of patterns of individual use and assignment of completed trials to individuals for error analysis without long hours of observation. The purpose of the present paper is to describe an automated system of individual identification that assigns an identification number to every completed trial. It should be noted that the identification methodology described is applicable to species other than those in the order Primates and to tasks other than joystick tasks.

An individually housed, adult male bonnet macaque was selected as the demonstration animal. It was reasoned that

This work was supported by Public Health Service Grant RR05321 and the State University of New York. The microchips ( $\$ 10$ each, loaded in a special syringe) and identity-tag reader and antenna $(\$ 1,250)$ used in this study were manufactured by AVID, 3179 Hamner Avenue, Suite 5, Norco, CA 91760 . Correspondence should be addressed to M. W. Andrews, Dept. of Psychiatry, Box 120, SUNY Health Science Ctr., 450 Clarkson Ave., Brooklyn, NY 11203. a system that could distinguish which arm was used by one subject would be able to distinguish among the arms of a number of different subjects. Therefore, the data presented in the present paper, obtained during the initial implementation of the system, refer to differentiation between arms of a single subject rather than differentiation among the arms of different subjects. The systematic study of handedness (see reviews in Fagot \& Vauclair, 1991; MacNeilage, 1991), although not a primary goal in the Primate Behavior Laboratory, is certainly an important potential application of the system.

\section{METHOD}

\section{Subject}

The male bonnet macaque used in this study was 15.5 years old and naive to all joystick tasks. The subject had ad-lib access to water and standard laboratory chow throughout the study.

\section{Apparatus}

The identification system is based on an implantable microchip identity tag and identity-tag reader manufactured by the AVID Company (Norco, CA). The identity tag is $14 \mathrm{~mm}$ long $\times 2 \mathrm{~mm}$ in diameter and weighs less than $0.1 \mathrm{~g}$; it is biologically inert, batteryfree, and designed to last the lifetime of the subject. The AVID Company preprograms each identity tag with a unique identification number. The identity-tag reader can be controlled, and identification numbers input, through the serial port of a computer, using 8 transmit bits and 2,400 baud. In addition to identification numbers in the format "AVID* $123 * 456 * 789$," status messages ("LOOKING," "No ID Found," and "AVID IS READY") are sent from the reader.

The subject was housed alone in a standard primate cage $(80 \mathrm{~cm}$ wide $\times 123 \mathrm{~cm}$ high $\times 88 \mathrm{~cm}$ deep). The front of the cage had been modified with a metal-framed, clear Plexiglas insert $(51 \mathrm{~cm}$ wide $\times 51 \mathrm{~cm}$ high) that replaced the stainless steel guillotine door. The insert consisted of a double layer of 6.4- $\mathrm{mm}$ Plexiglas. The bottom of the insert was $24 \mathrm{~cm}$ from the pen floor. A 6-cm-diam hole in the insert located $31.5 \mathrm{~cm}$ from the pen floor provided the subject with single-arm access to the equipment located outside the pen.

With the exception of the joystick and food-reward cup, the equipment located outside the monkey's pen was shielded by a clear Plexiglas screen attached to the cart on which the equipment was located. The essential components of the equipment were as follows: (1) a joystick mounted with the handle oriented horizontally, $8 \mathrm{~cm}$ from the hole in the cage insert; (2) a food-reward cup $(4.5 \mathrm{~cm}$ in 


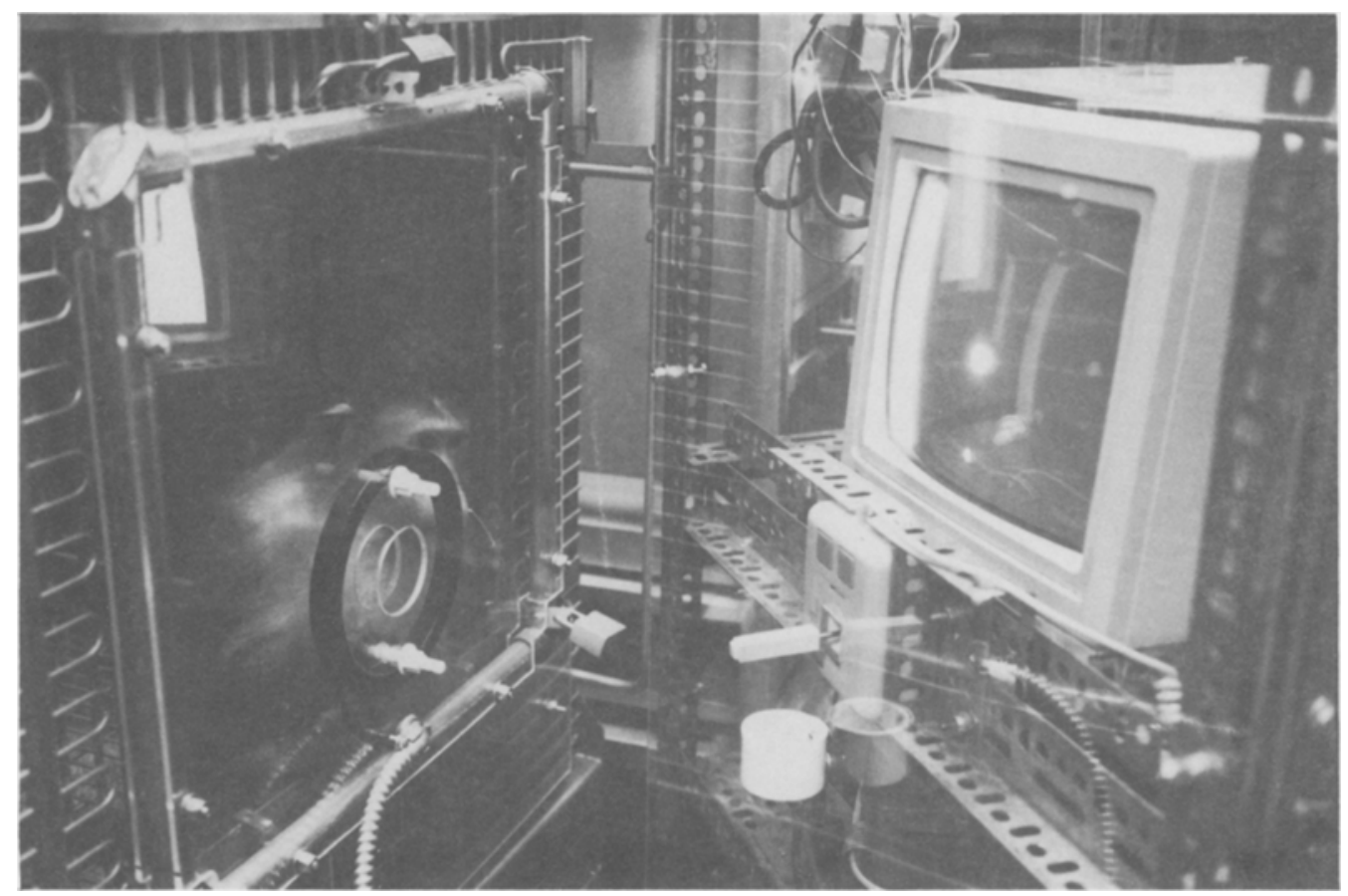

Figure 1. Photograph of the experimental equipment. The cart containing the joystick and monitor is shown pivoted out from the monkey's cage to illustrate the components.

diameter $\times 3.5 \mathrm{~cm}$ deep) located $5 \mathrm{~cm}$ directly below the joystick handle; (3) a PC/XT-compatible computer; (4) a 36-cm color VGA monitor, located immediately above and behind the joystick; (5) a pellet dispenser that delivered 190 -mg banana-flavored food pellets; (6) an identity-tag reader connected to the serial port of the computer; (7) a ring antenna $(15 \mathrm{~cm}$ in diameter $\times 1.5 \mathrm{~cm}$ thick), located between the layers of the cage insert and encircling the access hole, connected by cable to the identity-tag reader; and (8) software for the joystick task. The essential components of the system are shown in Figure 1.

Because the identity-tag reader senses variations in electromagnetic fields, it is subject to interference from computer monitors, large motors, and some kinds of switching power supplies. The Sceptre Model CT-6G monitor was found to work well, permitting an effective range for reading the microchip extending approximately $5 \mathrm{~cm}$ on either side of the antenna. Because metal objects absorb electromagnetic radiation, it is also important that the antenna be kept away from such objects to avoid reduction of the effective range for reading the microchip.

\section{Procedure}

Using a modified Monoject syringe with a 2-cm, 12-ga needle supplied by the AVID Company, a microchip was implanted subcutaneously in each forearm of the monkey under anesthesia. Each chip was implanted such that it would be in the plane of the antenna when the monkey grabbed the center of the joystick $(16 \mathrm{~cm}$ from the fingertips for the present apparatus design). The range of the antenna in reading the microchips, however, allowed a variation in grip, ranging from grasping the tip of the joystick with the fingertips to firmly grasping the base of the joystick.

The data presented in the present paper are from the initial training of the subject, in which the target size was reduced in stages from the entire border of the screen to one randomly selected side of the screen on each trial (top, bottom, left, or right). Contacting the target with the cursor completed a trial and initiated the following sequence of events: (1) The identity-tag reader was triggered to read an identification number; (2) an audible tone was sounded that served as a secondary reinforcer and occupied much of the necessary delay between triggering the identity-tag reader and inputting data from it; (3) the identification number was input into the computer; and (4) a treat was dispensed into the food-reward cup.

The total elapsed time from completion of a trial to arrival of a pellet in the reward cup was approximately $2.9 \mathrm{sec}$. With the audible tone as an immediate secondary reinforcer, this delay did not appear to retard learning. The 2.9 -sec delay resulted primarily from the time required for the electromechanical pellet-dispensing system to deliver a pellet into the reward cup approximately $75 \mathrm{~cm}$ away and from a 600-msec time interval imposed between triggering the reader and inputting data from it. A 100 -msec interval between the trigger pulse and inputting data has subsequently been found to be adequate.

\section{RESULTS}

No evidence of infection or irritation at the microchip implantation sites was observed; the monkey did not even attend to the implantation sites after the first day. Furthermore, despite thousands of reaches through a narrow opening, no damage occurred to the microchips. The implantation was not only safe, but was also well located; during observation by the author of a large number of trials over a period of weeks, the microchip was never observed to be out of the range of the antenna at the completion of a trial.

The identity-tag reader was highly reliable in reading and transmitting the identity-tag number. During hundreds 
of trials with different identity tags during development and testing of the system by the author and other laboratory personnel, no number was read incorrectly. Furthermore, every identification number recorded on over 6,600 trials by the monkey was one of the two implanted numbers.

The bonnet macaque male in the present study completed $99.7 \%$ of all trials using his left arm. This did not appear to be the result of differential sensitivity of the system to the microchips implanted in the left and right forearms. For right-arm trials, $68 \%$ (13) occurred in a rapid sequence with an average of only $6.3 \mathrm{sec}$ between them. The comparable values for the immediately preceding and immediately following left-arm trials were 9.2 and $7.1 \mathrm{sec}$, respectively.

\section{DISCUSSION}

The microchip identity tag was a safe and reliable means of identification of the arm used to perform a joystick task. Implantation of the microchip posed no threat to either the health or comfort of the subject animal following the implantation procedure. Furthermore, the effective range of the antenna ( $\pm 6 \mathrm{~cm}$ from the midline plane) was sufficient to ensure that the microchip was within the range of the antenna at the completion of all observed trials, despite natural variations in the monkey's grip. It should also be emphasized that preference for using the left arm was not the result of differential sensitivity of the system to the microchips implanted in the left and right forearms; in fact, for the only sequence completed with the nonpreferred right arm, the monkey completed trials at a slightly faster rate than for comparable trials completed with the preferred left arm.

In periodic ad-lib observations over several weeks, the subject never grasped the joystick with his right hand. It is only because of the power of this system to read the microchip number corresponding to the utilized arm on thousands of trials that any use of the right arm was ascertained. Together with the reliability of the identity-tag reader pointing to a low probability that the right-arm identification reflected a malfunction, this data clearly indicates the value of an automated identification system in the study of infrequent events.
The microchip-identity-tag system provides an important research resource. It is expanding the range of application of joystick tasks that have already demonstrated broad applicability; the identification system is now being implemented for determination of individual patterns of task engagement in social groups. Other possible applications of the system include tracking small animals with implanted microchips by requiring them to pass near or through the antenna, and using the identity tag for temporary, noninvasive identification by placing it in a removable collar or bracelet. The range of application of the identification system is limited only by the imagination of investigators.

\section{REFERENCES}

ANDREWs, M. W. (1993). Video-task paradigm extended to Saimiri. Perceptual \& Motor Skills, 76, 183-191.

ANDREws, M. W., \& Rosenblum, L. A. (in press). Developmental consequences of altered dyadic coping patterns in bonnet macaques. Proceedings of the 14th Congress of the International Primatological Society.

FAGOT, J., \& VAuClair, J. (1991). Manual laterality in nonhuman primates: A distinction between handedness and manual specialization. Psychological Bulletin, 109, 76-89.

MacNeilage, P. F. (1991). Patterns of handedness across the primate order. In A. Ehara, T. Kimura, O. Takenaka, \& M. Iwamoto (Eds.), Primatology today (pp. 275-278). Amsterdam: Elsevier.

Rosenblum, L. A., \& ANDrews, M. W. (in press). Environmental enrichment/psychological well-being of captive primates. In B. T. Bennet, C. Abee, \& R. Henrickson (Eds.), Nonhuman primates in biomedical research: Vol. 1. Biology and management. Orlando, FL: Academic Press.

WASHBURN, D. A. (1990). PC-compatible computer-generated stimuli for video-task testing. Behavior Research Methods, Instruments, \& Computers, 22, 132-135.

WASHBURN, D. A. (1992). Analyzing the path of responding in mazesolving and other tasks. Behavior Research Methods, Instruments, \& Computers, 24, 248-252.

Washburn, D. A., Hopkins, W. D., \& Rumbaugh, D. M. (1989). Automation of learning-set testing: The video-task paradigm. Behavior Research Methods, Instruments, \& Computers, 21, 281-284.

Washburn, D. A., \& Rumbaugh, D. M. (1992). Testing primates with joystick-based automated apparatus: Lessons from the Language Research Center's Computerized Test System. Behavior Research Methods, Instruments, \& Computers, 24, 157-164.

(Manuscript received April 26, 1993; revision accepted for publication August 19, 1993.) 